\title{
WAKE-UP REEEIVERS FOR WIRELESS SENSOR NETWORKS: BENEFITS AND CHALLENGES
}

\author{
ILKer Demirkol AND Cem ERSOY, BogaZiCI UNIVERSITY \\ ERTAN ONUR, DELFT UNIVERSITY OF TECHNOLOGY
}

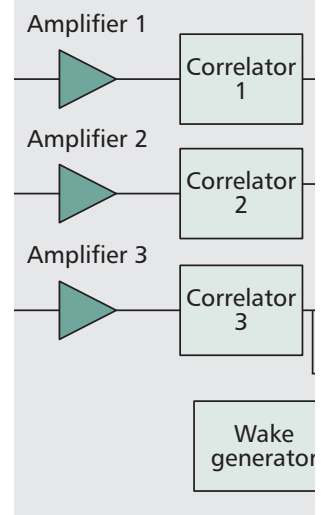

The authors

investigate the

benefits achieved

with wake-up

receivers along with

the challenges

observed. In addi-

tion, they present

an overview of

state-of-the-art

hardware and

networking protocol

proposals.

\begin{abstract}
For successful data delivery, the destination nodes should be listening to the medium to receive data when the sender node starts data communication. To achieve this synchronization, there are different rendezvous schemes, among which the most energy-efficient is utilizing wakeup receivers. Current hardware technologies of wake-up receivers enable us to evaluate them as a promising solution for wireless sensor networks. In this article the benefits achieved with wake-up receivers are investigated along with the challenges observed. In addition, an overview of state-of-the-art hardware and networking protocol proposals is presented. As wake-up receivers offer new opportunities, new potential application areas are also presented and discussed.
\end{abstract}

\section{INTRODUCTION}

Wireless sensor networks (WSNs) consist of autonomous nodes equipped with sensors to monitor physical or environmental conditions. The objective of a WSN is to sense the environment and communicate the collected information to the base station. Since energy is the bottleneck attribute of the WSNs, reducing redundant energy consumption is a significant research issue. Instead of sleep scheduling, ondemand or event-triggered operation of the network will improve the performance and the lifetime. This approach is viable with the wakeup receiver technology. In this article, we address the benefits and the challenges of utilizing the wake-up receivers in the WSN applications.

In general, the energy consumed for communication is dominant over that for computation or sensing activities of the sensors. Hence, the main objective in WSN research is to reduce unnecessary communication. An efficient protocol design must reduce the amount of communi-

This work is supported by TUBITAK under the grant number 106E082. cation while achieving the requirements of the network. The factors that impact the energy consumption of sensors in terms of communication are shown in Table 1.

One method to decrease the unnecessary energy consumption is to implement an energyefficient configuration protocol where sensors operate under a well defined sleep schedule. That is, inactive sensors switch to off mode or low-power listening mode. When two nodes are to communicate, the receiver node must be awake when the sender initiates the communication, which is referred to as rendezvous [1]. There are three types of rendezvous schemes:

- Pure synchronous rendezvous: The sensor clocks are presynchronized such that the wake-up time of each node is known a priori. This scheme requires recurrent time synchronization that consumes considerable energy. Moreover, the sensors wake up even if there is no packet to transmit or receive, which results in idle listening or overhearing.

- Pseudo-asynchronous rendezvous: Source nodes wake up and emit a preamble signal that indicates the intention of data transmission. The preamble time is long enough to coincide with the wake-up schedule of the destination node. Upon waking up and sensing the preamble, the destination node recognizes the intended packet transmission. In this scheme time synchronization is not required, but sensors still follow a duty cycle and consume considerable energy with preamble signaling.

- Pure asynchronous rendezvous: Sensors reside in deep sleep and can be woken up by their neighbors on demand with very low-power wake-up receivers. Whenever a node intends to send a packet, first it wakes up the destination node and then sends the packet. Therefore, wake-up receivers are a solution to the redundant energy consumption caused by rendezvous.

The current wake-up receiver hardware products on the market along with ongoing hardware research enable us to evaluate the wake-up receiver technique as a candidate feature for numerous WSN applications. In this article we 


\begin{tabular}{|l} 
- Data or packet redundancy such as correlated event detections \\
Application layer \\
- The messaging caused by application requirements such as software updates \\
- The messaging for monitoring activities such as health checking of the sensors
\end{tabular}

Table 1. The factors that impact the energy consumption of sensors categorized by the OSI layers.

investigate the wake-up receiver technology thoroughly, past, present, and future. After presenting the state of the art in wake-up technology, we analyze the benefits and challenges, and point out their application areas in the wireless sensor networks.

\section{THE STATE OF THE ART IN WAKE-UP RECEIVER RESEARCH}

Studies on wake-up receivers consist of two parts:

- Hardware development for low-power wake-up circuitry

- Software development for networking protocols that utilize wake-up receivers

The wake-up receiver technologies proposed in the literature or available on the market exhibit various working principles. We present the classification tree of wake-up receiver technologies that are applicable to both hardware and software in Fig. 1. For the hardware proposals and products, this classification represents the features offered, whereas for the protocol studies, this tree represents the hardware features utilized.

Based on their energy sources, wake-up receiver proposals can be classified as passive wake-up receivers in which the wake-up circuitry is triggered by an external energy source and as active wake-up receivers in which the internal battery is used such that the wake-up receiver monitors for the possible wake-up signal. The channel on which the wake-up signal is sent can be the same as the main radio communication channel (i.e., shared channel), or a separate channel can be used for the wake-up signaling. This separate wake-up channel may consist of multiple channels to be able to wake up specific nodes utilizing frequency division. Although a separate channel increases the cost and complexity of the sensor node, as indicated in [2], the penalty for channel addition is minimal, as the radio typically accounts for less than 15 percent of the cost of a sensor node.

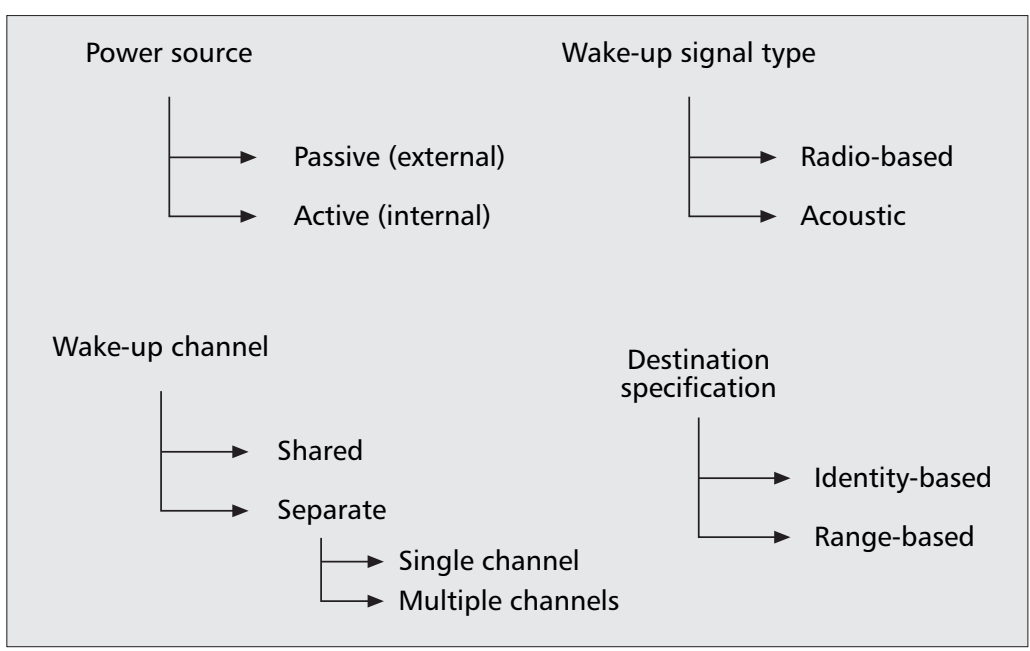

Figure 1. Classification of wake-up receiver schemes.

The wake-up signal can be a single wake-up tone or a bit sequence. If all the nodes that receive the tone wake up, the scheme is referred to as range-based wake-up, which is appropriate for multicasting. On the other hand, the wake-up signal may consist of a bit sequence to address the destination. After the reception of a wake-up signal, nodes check if the bit sequence refers to them; if so, the destination wakes up. This scheme is referred to as identity-based wake-up and allows unicasting.

Radio signals are used as wake-up signals in radio-based wake-up receivers, or wake-up radio for short. Alternatively, acoustic wake-up receivers are triggered by acoustic signals (i.e., external sounds). When the observed level of the external sound reaches a threshold, the wake-up circuitry is activated.

\section{Hardware Level Proposals}

Commercialized wake-up receivers that can achieve low energy consumption by using an active wake-up receiver exist. For instance, a 
As the wake-up

hardware improves

and generates a

promising solution

for energy-efficient

communication in

wireless sensor

networks, related

communication

protocols are also

enhanced.

\begin{tabular}{lll}
$\begin{array}{l}\text { Operating } \\
\text { mode }\end{array}$ & $\begin{array}{l}\text { Regulator on, } \\
\text { VCC }=3 \mathrm{~V}\end{array}$ & $\begin{array}{l}\text { Regulator off, } \\
\text { VCC }=2.4 \mathrm{~V}\end{array}$ \\
\hline Sleep & $0.8 \mu \mathrm{A}$ & $0.3 \mu \mathrm{A}$ \\
\hline Standby & $7.0 \mu \mathrm{A}$ & $6.5 \mu \mathrm{A}$ \\
\hline Receive & $7.2 \mu \mathrm{A}$ & $6.8 \mu \mathrm{A}$ \\
\hline
\end{tabular}

Table 2. Current consumption values for a wake-up receiver on the market [3].

wake-up receiver with three channels is available on the market whose simplified block diagram is shown in Fig. 2. It has three power management modes: sleep, standby, and receive [3]. Table 2 presents the currents drawn at each mode. In sleep mode, all channels are switched off. In standby mode, selected channels are switched on and ready to receive data. The amplifier of the selected channel is on, whereas the correlator is powered down as long as no input signal is detected at the input. The enabled channel switches from standby mode to receive mode when the input signal is detected and stays in the receive mode as long as the input signal is detected. In receive mode, the correlator of the channel is active and scans the input signal waveform for a valid wake-up pattern. The channel goes back to standby mode after an adjustable timeout period if no input signal is detected. An optional regulator adjusts the internal voltage to $2.4 \mathrm{~V}$. The current values presented in Table 2 are for the case where all three channels are enabled. Further reduction can be achieved with fewer channels. This wakeup receiver is triggered with a specific bit sequence; however, the wake-up pattern is not adjustable, and hence only range-based wake-up can be achieved.

Hardware proposals that are based on the super-regenerative principle are also available [4, 5]. The super-regenerative receivers use a second lower frequency oscillator to provide single device circuit gains of six orders of magnitude. This second oscillation periodically interrupts the main radio frequency (RF) oscillation, allowing the RF signal to be built up continually. Hence, they enable very low-power active wakeup receivers. An earlier work by Joehl et al. presents an implementation of a super-regenerative transceiver that consumes $3.6 \mathrm{~mW}$ for a receiver sensitivity of $-105 \mathrm{dBm}$, and the emitter current consumption is $6 \mathrm{~mA}$ for $0 \mathrm{dBm}$ output power [4]. Recently, a super-regenerative transceiver that consumes $400 \mu \mathrm{W}$ on reception and $1.6 \mathrm{~mW}$ on transmission is proposed for wireless sensor networks by Otis et al. [5].

An important problem of low-power wakeup receivers is the false alarms caused by environmental noise. To overcome this difficulty, a wake-up receiver with three RF stages is proposed in [6]. A simplistic stage consuming around $\mathrm{nWs}$ is always active. On receiving a signal, it activates a more complex stage consuming in $\mu \mathrm{Ws}$ to check if the received signal is a specific wake-up code. Only if this stage confirms a valid wake-up condition, the main transceiver is activated. Specific patterns of signals are used in a shared channel for wake-up signaling.

$\mathrm{Gu}$ et al. propose a novel approach in which the wake-up receiver is activated by the energy of the received radio signals [7]. This type of wake-up receiver can be categorized as passive since it does not require additional power. To trigger the receiver, a period of time is required to collect adequate energy that is not negligible. The introduced wake-up delay is proven analytically to be dependent on the wake-up range (i.e., the distance to the target node) [7]. For instance, $55 \mathrm{~ms}$ delay is needed to wake up a node that is $30 \mathrm{~m}$ away. The passive wake-up circuitry is not totally battery-free; the comparators and amplifiers consume energy. Fortunately, the negligible current consumptions of the comparator and amplifier are $350 \mathrm{nA}$ and $880 \mathrm{nA}$, respectively. The wake-up signals should be sent at a special radio frequency so that normal radio communication does not unintentionally wake up the idle part of the network. In addition, the authors present an identity-based wake-up with the help of multiple transceivers with different frequencies. The combination of frequencies are assigned to the neighbor nodes so that when a transmission is done with a set of frequencies, only the corresponding nodes wake up.

Other than RF wake-up receivers, an acoustic wake-up receiver is proposed in [8]. The acoustic wake-up receiver consumes around $835 \mathrm{nW}$. Experiments are done with sounds generated by external devices, and the level of the sound determines the wake-up range. This technology can be improved to be embedded in sensor nodes. Xbow Mica motes are equipped with a microphone that can be used as an acoustic wake-up receiver, since a sounder also exists on these nodes. Table 3 categorizes hardware proposals for the wake-up receivers based on the classification given in Fig. 1.

\section{Software Level Proposals}

As wake-up hardware improves and generates a promising solution for energy-efficient communication in WSNs, related communication protocols are also enhanced. In this section these studies are overviewed based on the classification presented in Fig. 1.

\section{Communication Protocols for Active Wake-Up Receivers} - A topology management technique that utilizes a wake-up radio is proposed in [2], Sparse Topology and Energy Management (STEM). The aim of the wake-up radio is to separate data communication from the preamble messaging used for synchronization of the sender and receiver. Energy saving is achieved by introducing latency at the synchronization of the nodes via the wake-up radio. Even if the wake-up radio is not ultra-low-power, energy savings can be achieved with this method. Two variants exist. In STEM-B a beacon is sent that includes the identity of the sender and the destination node, whereas in STEM-T, a busy tone is sent which wakes every node that hears the tone. STEM-B therefore utilizes an identity-based wake-up receiver, whereas STEM-T utilizes a range-based wake-up receiver. 


\begin{tabular}{|lllll|}
\hline Hardware & Power source & Signal type & Wake-up channel & $\begin{array}{l}\text { Destination } \\
\text { specification }\end{array}$ \\
\hline AS3931 [3] & Internal & Radio-based & Separate (multichannel) & Range-based \\
\hline Otis et al. [5] & Internal & Radio-based & Shared/separate & Range-based \\
\hline Mark et al. [6] & Internal & Radio-based & Shared & Range-based \\
\hline Gu et al. [7] & External & Radio-based & Separate (multichannel) & Identity-based \\
\hline Goldberg et al. [8] & Internal & Acoustic & Separate & Range-based \\
\hline
\end{tabular}

Table 3. Categorization of wake-up receiver hardware proposals.

Similarly, in [9] a wake-up receiver with duty cycle is used that utilizes preamble-based synchronization between the sender and the receiver. In addition to wake-up with preamble messaging, a triggered wake-up is also defined in which every node wakes up once at duration $T$. The authors try to optimize the values of $T$ based on the given packet arrival rate with the aim of minimizing the total energy expenditure.

Since the wake-up delay increases the end-toend delay of data packets, a number of protocols are proposed to decrease the wake-up delay incurred. Latency Minimized Energy Efficient MAC (LEEM) is one such protocol and is a hop-ahead reservation scheme [10]. The idea is to reserve the next hop's channel, that is, to wake up the destination's next hop in advance.

Another alternative to STEM is Pipelined Tone Wake-Up Scheme (PTW) [11]. Again, a wake-up receiver has a certain duty cycle. Rangebased signaling is applied as in STEM-T. Additionally, in PTW the node that is receiving a data packet wakes up its neighbors for the next data transmission as in LEEM.

Wake-up receivers can be used for collisionfree MAC-layer communication as utilized in [12]. Although a routing protocol is proposed, the underlying MAC protocol is defined to send a wake-up signal on a broadcast channel. The address of the destination node is modulated with the wake-up. Access to the broadcast channel is CSMA/CA. A similar approach is applied in [13], where the authors propose a MAC protocol that combines CSMA and code-division multiple access (CDMA) techniques. The wakeup receiver used in [12] is identity-based, whereas the one used in [13] is range-based.

A wake-up channel is used in [14] as a control channel where request/clear to send (RTS/CTS)-like messaging and busy-till information can be exchanged. The authors compare this protocol to S-MAC and achieve nearly 66 percent reduction in energy consumption and 33-60 percent better end-to-end delay values.

The wake-up receiver idea is also proposed for other wireless networks. For instance, in [15] the lifetime of GSM devices is improved using a low-power control channel on a wireless LAN (WLAN). Experimental results show that the battery lifetime of these devices can be improved by 115 percent with this methodology.

Lin et al. propose two asynchronous scheduling methods and compare their performance results with the case of utilizing wake-up radio [1]. They derive a lower bound for the energy consumption of the wake-up radio which is achieved by setting the probability that the sender node will estimate the destination node's wake-up time within a specific time threshold to 1. A similar performance comparison between a wake-up receiver and a duty-cycled radio receiver is presented in [16].

In [17] analytical bounds on the lifetime of WSNs are studied. Authors utilize a wake-up radio in such a way that when a sensor wakes up and the channel is not free, it spends the awake time switching to a low-power wake-up radio. When the channel is free, the sensor powers up its main radio to communicate.

Song et al. utilize a range-based wake-up receiver [18] for a target tracking application on WSNs. The node that detects the target first wakes up all its neighbors to sense the target and announce their observations.

Various proposals presented in this section assume active wake-up receivers that require internal sources of energy. However, passive wake-up receivers that obtain their main energy from external sources are also utilized for the software proposals.

\section{Communication Protocols for Passive Wake-Up Receivers} - Khalil et al. propose Sleep-Wake Aware Local Monitoring (SLAM) [19] as a security mechanism for malicious nodes in which a passive wake-up receiver is utilized such as the one proposed in [7]. There are guard nodes that monitor the communications and try to figure out the malicious nodes. Each node awakens the guard nodes responsible for local monitoring of its next hop before communicating with it. They show that by utilizing wake-up receivers, the same level of security could be achieved, while the total energy consumed is decreased by $66-90$ percent.

Table 4 categorizes protocol studies that utilize wake-up receivers based on the classification given in Fig. 1.

\section{Benefits, Challenges, and Trade-OFFs}

In this section we present the benefits achieved with utilization of wake-up receivers and the challenges observed in wake-up-receiver-based WSNs. These challenges should be studied carefully for the necessary solutions to be able 


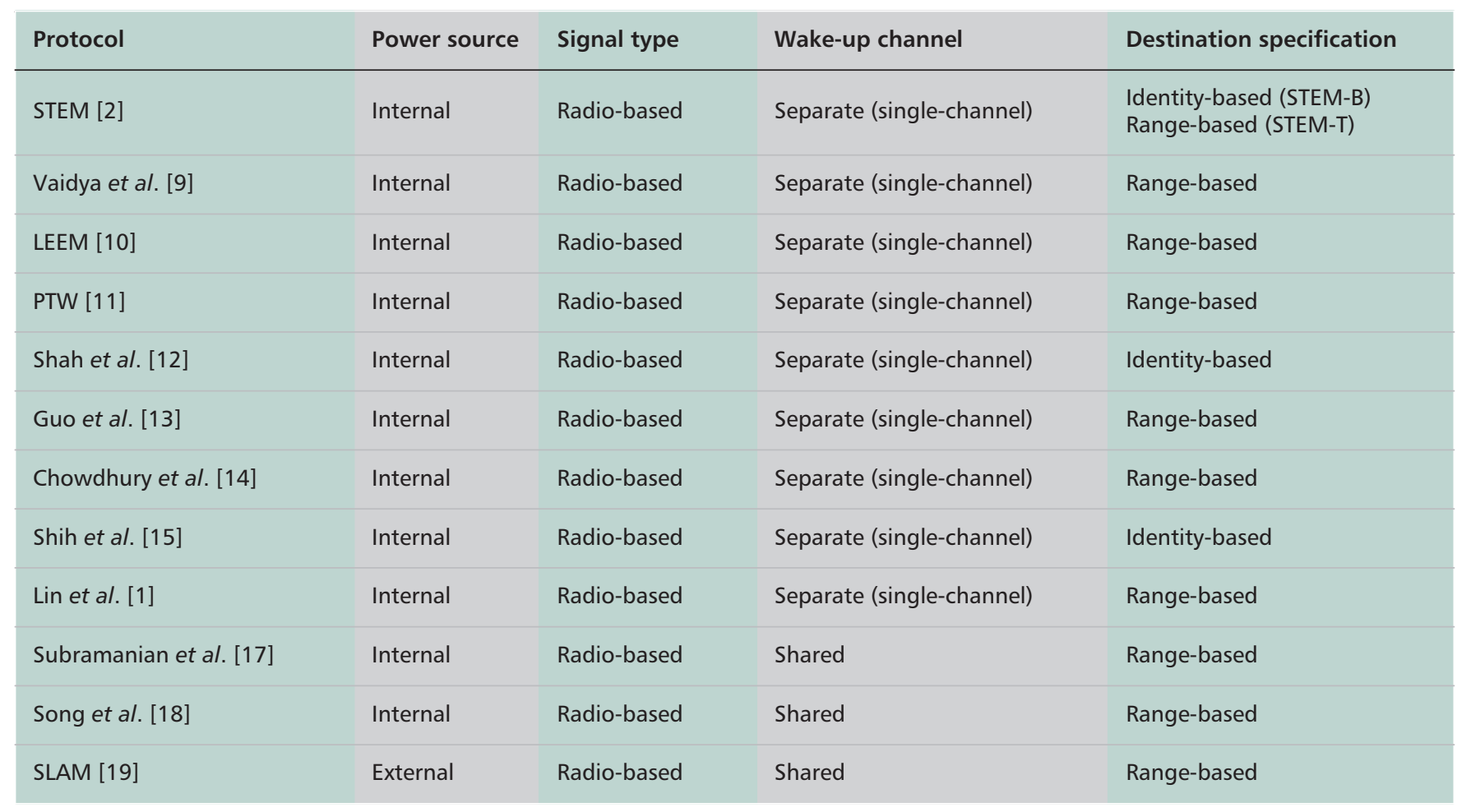

Table 4. Categorization of wake-up-receiver-based protocol research.

to take advantage of the offered benefits. Moreover, the trade-offs observed in networks with wake-up receivers are stated. Each of these trade-offs requires detailed analysis to enable the discovery of the optimum network settings.

\section{BENEFITS}

Energy-Conservation - A dominant energy waste observed in WSN applications is idle listening, which occurs because of periodic wake-ups regardless of the data communication needed. The wake-up strategy should be energy efficient [20]. The wake-up receiver presents an energy-efficient solution to idle listening as the nodes only wake up when there is a message intended for them. Chowdhury et al. show that the energy consumption of S-MAC can be reduced significantly using wake-up receivers [14]. Since active wake-up receivers are also an energy consumption source for sensor nodes, they should be ultra-low-power to acquire the energy gain.

Minimum Overhead - As the wake-up receiver presents a purely asynchronous rendezvous scheme, the overhead incurred by time synchronization of the two other rendezvous schemes are dissipated. This overhead causes higher energy consumption, as shown by $[1,16]$. The communication overhead to form the routing backbone also becomes redundant as each node wakes up its relay. For instance, Dhanaraj et al. propose a method where a destination node first wakes up its relay before receiving the data intended for it [10]. In addition, instead of having clusters with regular duty cycles, formation of clusters can be done only with event occurrences.
Different Forms of Use - For efficient use of a WSN for a target application, wake-up receiver technology enables various new methods such as the on-demand target monitoring method proposed in [18] where a node that detects a target wakes up all its neighbors to receive their observations about the target. The next section presents application areas for wake-up receiver technology.

Dynamic Behavior - System-wide parameters create inefficiencies as the network load differs based on location. Hence, parameters such as the duty cycle and preamble length should be dynamically set based on localized requirements. Since a wake-up receiver enables dynamic duty cycling and dynamic communication rendezvous, several inefficient parameter definitions become unnecessary, which results in a more efficient network, as shown by $[1,16]$.

Overhearing Is Decreased - With the help of identity-based wake-up receivers, overhearing is reduced since only the destined nodes will wake up and listen to the medium, as in $[2,15]$.

\section{CHALLENGES}

Cost and Hardware Complexity - If a wake-up radio utilizes separate channel(s), the hardware complexity and cost of sensor nodes will increase. This is especially important when multiple wakeup channels are employed, as in [3, 7]. However, as indicated in [2], the radio typically accounts for less than 15 percent of the cost of a sensor node.

Delay - With a passive wake-up radio, such as the one proposed in [7], wake-up delay is incurred, which may become significant for the 
requirements of the application. The impact of the end-to-end delay may be significant when the number of hops to the sink is large. For applications such as target tracking, point-topoint (one-hop) delay is critical for the performance of the application.

Topology Change - Since the wake-up range may not be the same as the communication range of the main radio, topology for the wake-up receivers can be different than the actual network topology. The neighborhoods achieved with wake-up receivers hence result in a topology different from the original network topology. The former may result in a disconnected network, although the latter is connected. In such circumstances the problem should be realized by the protocols, and necessary solutions should be applied. In addition, even if the wake-up receivers form a connected network, the number of hops on the routing path may increase if the wake-up range is less than the communication range, which can cause inefficient routing of the packets. Unfortunately, there is no work in the literature yet that investigates the effect of separate topology caused by wake-up receivers.

Energy Hole Problem - Ahmed et al. define the hole problem as the result of some anomalies in WSNs that impair the functionality of the network [21]. Specifically, the coverage hole is defined as the area not covered by any sensor due to anomalies such as random deployment creating voids, node failures, or jamming. If the wake-up range is smaller than the communication range of the main radio, the topology of the network formed by the wake-up receivers may contain more or larger energy holes. This may result in inefficient network utilization; for instance, passing over the larger energy holes requires more data communication and hence more energy.

Overhearing Increases for Shared-Channel and RangeBased Wake-Up Receivers - For range-based wakeup schemes, all nodes hearing the tone switch to active state. Since all surrounding nodes will wake up at each transmission, overhearing can be increased compared to other protocols. To prevent overhearing, identity-based wake-up receivers can be used as in $[2,15]$.

\section{TRADE-OFFS}

The following trade-offs are expected in networks where wake-up receivers are used. Although the quantification of these trade-offs requires detailed performance evaluation studies, no work on this topic has appeared in the literature yet.

Wake-Up Range vs. Energy Consumption - As the density of sensor deployment increases, the distances among sensors become shorter. For dense deployments, multihop wake-up may be possible. In the multihop wake-up scheme, a sensor node triggers a node that is several hops away in the communication (routing) graph. For sparse deployments, the wake-up range affects energy consumption. The range for a wake-up radio can be adjusted with the energy consumption trade- off. Distant sensors can be awakened by consuming more energy. Determining an efficient wakeup distance depends on design decisions such as the applied routing strategy.

Wake-up Range vs. Delay - Multihop communication is common in WSNs. Hence, the end-to-end reporting delay becomes a critical metric for performance evaluation. Increasing the wake-up range decreases the overall hop count, which in turn decreases the delay.

In-Band or Out-of-Band Wake-Up Radio - The same channel(s) as that of the communication stack can be used for a wake-up radio. This reduces the implementation costs, but has lower resistance to jamming attacks to the communication stack and increases the probability of collision. If separate channels are utilized, the costs increase; however, resistance to jamming attacks increases, and energy consumption due to overhearing decreases.

\section{ApPLICATION Areas}

When WSNs are categorized according to the mode of data collection, two main approaches are studied in the literature. These are eventdriven and periodic (or query-based) data collection. In this section we discuss how a wake-up radio can be used for these application categories.

\section{EVENT-DRIVEn ApPLICATIONS}

In this category sensors inform the sink of their decisions when a specific event occurs. Typical examples are a forest fire detection system, sniper localization, or a surveillance system whose duty is intrusion detection, target tracking, or perimeter protection in scenarios such as border monitoring against penetration by hostile elements. The events in a WSN application can be spatiotemporally correlated. For example, in a surveillance application, if a sensor detects a target, it is highly probable that another sensor at about the same distance will also detect the same target. Furthermore, these detections will occur at about the same period of time. Due to redundant deployment, the impact of the spatial and temporal correlations can be very influential on energy consumption. Instead of centralized decision fusion, a local subset of sensors may collaborate to decide on the event using data/ information fusion techniques. If the sensors are equipped with wake-up circuitry, a dynamic clustering mechanism can be viable instead of assigning sensors to clusters prior to network operation. Sensors that are spatially collocated with the event can form a cluster dynamically. This scheme may not only yield better accuracy, but also consume less energy.

For event-driven applications, the adaptivity of the network to some attributes of the event, such as its location, suggests developing crosslayer protocols with the sensing circuitry. This is a challenging research topic that needs detailed analysis. For example, based on the location of the event, the routing strategy may be altered. In a surveillance application, assume an intruder with a jammer. The sensor that detects the
Multi-hop communi-

cation is common in

WSNs. Hence, the

end-to-end reporting

delay becomes a

critical metric for the

performance

evaluation. Increasing the wake-up range, decreases the overall hop count which in turn decreases the delay. 


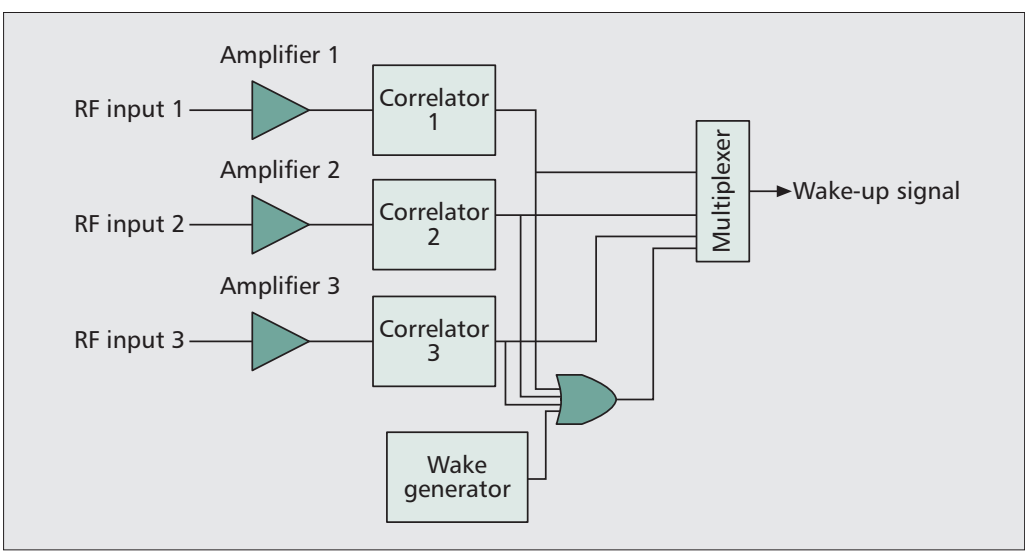

- Figure 2. Simplified block diagram for a wake-up receiver on the market [3].

intruder may apply a routing strategy to minimize the effect of the jammer by waking up the sensors that are far enough from the intruder.

For target tracking or sniper localization scenarios, sensors that detect an event may trigger the surrounding nodes, and a locally activated set of sensors may track/localize the target. Activating only the sensors close to the path followed by the target is a reasonable approach. The awakened group of sensors may localize the target more accurately. In surveillance applications, the frontier sensors may invoke some set of valuable or costly sensors behind the perimeter to produce a barrier to reduce the false alarms. This scheme suggests a layered topology where overlays are probable.

The interaction of the wake-up receivers with the sensor node and with the network for a simple event-driven application is shown in Fig. 3 based on a target detection scenario. The identity-based wake-up scheme enables point-to-point communication. While waking up the neighbors, it is possible to communicate some valuable information. In the surveillance scenario shown in Fig. 3, the sensor that detects a target may communicate the type of target (e.g., a soldier or an armored military vehicle), which helps the awakened sensor decide which sensing circuitry to activate. Such an application is reasonable if the sensing circuitry consumes comparable energy to the radio subsystem. For example, micro impulse radar sensors spend a couple of orders higher energy than passive infrared sensors. In this scenario, when the first sensor detects the target, its sensing circuitry triggers the wake-up receiver. First, a burst of signal is sent to the next hop (second sensor) to trigger the first stage of the wake-up receiver. Afterward, the identity of the second sensor and the target type are sent. The second stage of the wake-up receiver of the second sensor turns on the main radio if the sent identity matches itself. Then the main radios can communicate, and the second sensor may turn on the neccessary sensing circuitry based on the type of target.

A similar scenario can be devised to consider the preservation of the sensing coverage in case of sensor failures using the redundancy of the deployment. Assume that each sensor is assigned one or more standby buddy sensors. When the battery level of the active sensor drops below a certain threshold, it can wake up one of its buddies to monitor the region. Two or more sensors that monitor almost the same area can be matched to act as the backup. This scheme increases the high availability of the surveillance network through active-standby operation. A similar scenario is presented in [20].

In an event-driven application, depending on the rate of events, utilization of the wake-up technology may become useless. If events occur frequently, some set of sensors have to sleep and wake up frequently. Hence, for such scenarios switching to periodic sleep scheduling is wiser. An example scenario can be the surveillance of museums. Consider visitors of a historic arena. The objective of the WSN is to detect the visitors who are trying to enter unallowed parts of the arena. During the open hours of the museum, the rate of these events may be larger than during the night shifts. Daytime operation of the network can be based on periodic sleep scheduling, whereas night time operation can follow the wake-up scheme. Rigorous analysis of when to switch from a wake-up scheme to sleep scheduling is required when such bimodal operation is probable.

\section{PERIOdIC OR QueRY-BASED APPLICATIONS}

Periodic or query-based data collection is common when environmental properties such as temperature or humidity levels of a field are to be monitored. For such applications, wake-up circuitry may be utilized to dismiss the time synchronization requirement. Instead of scheduled operation, a single sensor can manage the timing, and when the data is to be collected, it initiates communication by waking up its neighbors. Hence, an individual sensor may create a dynamic data flooding scheme where the data can be aggregated only by the awakened sensors. For example, the maximum value of temperature readings can be calculated as the data flows to the sink through the dynamically determined route to the sink. Based on the residual energy levels of individual sensors, the sensors may reject waking up. If a handshaking protocol is utilized for the wake-up stage, the sensors may dynamically alter their route based on their impacts on their neighbors. For example, during the handshake process, residual energy levels can be exchanged to minimize the effect of energy consumption on the relay functionality.

Assume that a WSN is deployed to monitor the depletion of minerals in soil where several types of crops are cultivated. The farmer requires the average level of mineral ratios from a portion of the field where only one type of crop is nurtured. To obtain this information, the WSN must be queried based on the location of sensors. This type of sensor network can be regarded as a distributed database. For such applications, the dissemination of queries requires intelligent routing techniques where wake-up circuitry can be used. For example, in this farming scenario the sensors located in the region of interest can be awakened. The medium access layers can be coordinated during the wake-up stage to avoid collisions during communication. Some packet transmission schedule for the specific event can be delivered to the surrounding nodes within the wake-up code. For example, SMAC can be modified to 


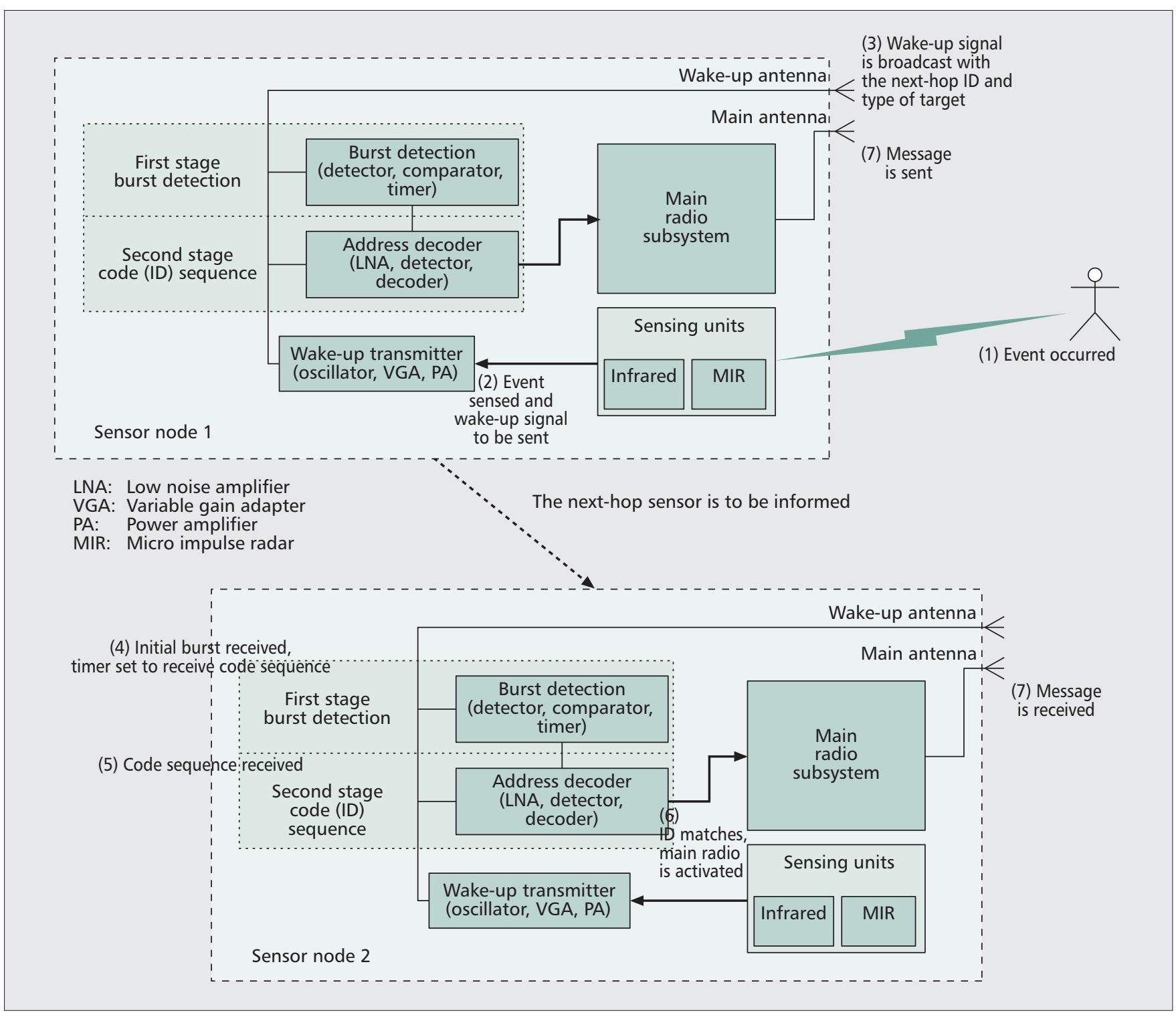

Figure 3. Interaction of wake-up receivers with the sensor node and with the network for a simple event-driven application. The sensor node that detects a target wakes up the next-hop sensor node. The wake-circuitry is simplified from [6].

distribute the schedules when an event is detected. This minimizes the energy consumption for duty scheduling.

\section{CONCLUSION}

The wake-up receiver technology presents a promising solution for energy-efficient rendezvous between sensor nodes. In this article a classification scheme is presented for wake-up receiver studies. The state-of-the-art wake-up receiver hardware and networking protocol proposals are investigated based on this classification scheme. The benefits of wake-up receiver use are presented along with the challenges that must be solved for its successful operation. In systems with wake-up receivers, a number of trade-offs are observed that have to be addressed for efficient network operation. These trade-offs are also listed and discussed. The new WSN application possibilities made available with this new technology are presented.

\section{REFERENCES}

[1] E. Y. A. Lin, J. M. Rabaey, and A. Wolisz, "Power-Efficient Rendezvous Schemes for Dense Wireless Sensor Networks," Proc. IEEE ICC, vol. 7, June 2004, pp. 3769-76.

[2] C. Schurgers et al., "Optimizing Sensor Networks in the Energy-Latency-Density Design Space," IEEE Trans. Mobile Comp., vol. 1, no. 1, Jan./Mar. 2002, pp. 70-80.

[3] Austria Microsystems, "3D Low Power Wakeup Receiver-AS3931," 2009; http://www.austriamicrosystems. com/eng/content/view/full/544

[4] N. Joehl et al., "A Low-Power 1-GHz Super-Regenerative Transceiver with Time-Shared PLL Control," IEEE J. SolidState Circuits, vol. 36, no. 7, July 2001, pp. 1025-31.

[5] B. Otis, Y. H. Chee, and J. Rabaey, "A 400mW-RX 1.6mW-TX Super-Regenerative Transceiver for Wireless Sensor Networks," IEEE ISSCC '05, Feb. 2005, pp. 396-7.

[6] S. von der Mark et al., "Three Stage Wakeup Scheme for Sensor Networks," SBMO/IEEE MTT-S Int'I. Conf. Microwave and Optoelectronics, July 2005, pp. 205-8.

[7] L. Gu and J. A. Stankovic, "Radio-Triggered Wake-up for Wireless Sensor Networks," Real-Time Sys., vol. 29, no. 2-3, 2005, pp. 157-82.

[8] D. H. Goldberg et al. "VLSI Implementation of an Energy-Aware Wake-Up Detector for an Acoustic Surveillance Sensor Network," ACM Trans. Sensor Net., vol. 2, no. 4, 2006, pp. 594-611.

[9] M. J. Miller and N. H. Vaidya, "A MAC Protocol to 
Reduce Sensor Network Energy Consumption Using a Wakeup Radio," IEEE Trans. Mobile Comp., vol. 4, no. 3. May/June 2005, pp. 228-42.

[10] M. Dhanaraj, B. S. Manoj, and C. S. R. Murthy, "A New Energy Efficient Protocol for Minimizing Multi-Hop Latency in Wireless Sensor Networks," Proc. 3rd IEEE PERCOM '05, Washington, DC, 2005, pp. 117-26.

[11] X. Yang and N. H.Vaidya, "A Wakeup Scheme for Sensor Networks: Achieving Balance between Energy Saving and End-to-End Delay," Proc. 10th IEEE RTAS '04, May 2004, pp. 19-26.

[12] R. C. Shah and J. M. Rabaey, "Energy Aware Routing for Low Energy Ad Hoc Sensor Networks," Proc. IEEE WCNC, vol. 1, Mar. 2002, pp. 350-55.

[13] C. Guo, L. C. Zhong, and J. Rabaey, "Low Power Distributed MAC for Ad Hoc Sensor Radio Networks," Proc. IEEE GLOBECOM, vol. 5, San Antonio, TX, Nov. 2001, pp. 2944-48.

[14] K. Chowdhury et al. "CMAC - A Multi-Channel Energy Efficient MAC for Wireless Sensor Networks," Proc. IEEE WCNC, vol. 2, Apr. 2006, pp. 1172-77.

[15] E. Shih, P. Bahl, and M. J. Sinclair, "Wake on Wireless: An Event Driven Energy Saving Strategy for Battery Operated Devices," Proc. ACM MobiCom, Sept. 2002, pp. 160-71.

[16] L. Zhong, J. M. Rabaey, and A. Wolisz, "An Integrated Data-Link Energy Model for Wireless Sensor Networks," Proc. IEEE ICC, vol. 7, June 2004, pp. 3777-83.

[17] R. Subramanian and F. Fekri, "Sleep Scheduling and Lifetime Maximization in Sensor Networks: Fundamental Limits and Optimal Solutions," Proc. 5th IPSN '06, Apr. 2006, pp. 218-25.

[18] L. Song and D. Hatzinakos, "A Cross-Layer Architecture of Wireless Sensor Networks for Target Tracking," IEEE/ACM Trans. Net., vol. 15, no. 1, Feb. 2007, pp. 145-58.

[19] Khalil, S. Bagchi, and N. B. Shroff, "SLAM: Sleep-Wake Aware Local Monitoring in Sensor Networks," Proc. 37th IEEE/IFIP Int'I. Conf. Dependable Sys, and Net. 2007, pp. 565-74.

[20] A. Boukerche, X. Fei, and R. B. Araujo, "An Optimal Coverage-Preserving Scheme for Wireless Sensor Networks Based on Local Information Exchange," Comp. Commun., vol. 30, no. 14-15, 2007, pp. 2708-20.
[21] N. Ahmed, S. S. Kanhere, and S. Jha, "The Holes Problem in Wireless Sensor Networks: A Survey," Mobile Comp. Commun. Review, vol. 9, no. 2, 2005, pp. 4-18.

\section{BIOGRAPHIES}

ILKER DEMIRKOL [M] (ilker@boun.edu.tr) received his B.Sc (with honors), M.Sc., and Ph.D. degrees in computer engineering from Bogazici University, Istanbul, Turkey, in 1998 2002, and 2008, respectively. Currently, he is a post-doctoral researcher at the University of Rochester, New York. He worked as a database, system, and network engineer from 1997 to 2004 . He was a research and teaching assistant in the Bogazici University Computer Engineering Department from 2004 to 2008. His research interests include the areas of wireless communications, wireless ad hoc and senso networks, and optimization of communication networks.

ERTAN ONUR [M] (onure@ieee.org) received a B.Sc. degree in computer engineering from Ege University, Izmir, Turkey, in 1997, and his M.Sc. and Ph.D. degrees in computer engineering from Bogazici University in 2001 and 2007, respectively. He is a BAL'93 graduate. After his B.S. degree, he worked for LMS Durability Technologies GmbH, Kaiserslautern, Germany. While working on his M.Sc. and Ph.D. degrees, he worked as a project leader at Global Bilgi, Istanbul, and as an R\&D project manager at Argela Technologies, Istanbul. Presently, he is an assistant professor at EEMCS, WMC, Delft University of Technology, the Netherlands. His research interests are in the area of computer networks, personal networks, and wireless and sensor networks.

CEM ERSOY [SM] (ersoy@boun.edu.tr) received his B.S. and M.S. degrees in electrical engineering from Bogazici University in 1984 and 1986, respectively. He worked as an R\&D engineer at NETAS A.S. between 1984 and 1986 . He received his Ph.D. in electrical engineering from Polytechnic University, Brooklyn, New York, in 1992. Currently, he is a professor in the Computer Engineering Department of Bogazici University. His research interests include performance evaluation of communication networks, wireless sensor networks, and mobile applications. He is the chairman of the IEEE Communications Society Turkish Chapter. 